Pacific Journal of Mathematics

SEPARATION PROPERTIES AND EXACT RADON-NIKODÝM
DERIVATIVES FOR BOUNDED FINITELY ADDITIVE

WAYNe C. Bell AND John William Hagood 


\title{
SEPARATION PROPERTIES AND EXACT RADON-NIKODYM DERIVATIVES FOR BOUNDED FINITELY ADDITIVE MEASURES
}

\author{
Wayne C. Bell and John W. Hagood
}

\begin{abstract}
Necessary and sufficient conditions for a $\mu$-continuous, bounded finitely additive measure to have an exact Radon-Nikodym derivative are obtained in terms of a new separation property intermediate between disjointness and mutual singularity.
\end{abstract}

1. Introduction. In the classical Radon-Nikodym theorem, it is proved that for countably additive real valued measures on a $\sigma$-field of sets, the class of measures that are absolutely continuous with respect to a fixed measure $\mu$ is precisely the set of measures having a representation of the form $\int f d \mu$ for a $\mu$-integrable function $f$. For bounded finitely additive measures on a field, these sets do not in general coincide. In this setting Radon-Nikodym theorems can have one of two goals: to characterize the absolute continuity class of $\mu$ or to provide necessary and sufficient conditions for a measure to have an exact Radon-Nikodym derivative with respect to $\mu$. In the history of the Radon-Nikodym theorem for bounded finitely additive scalar measures, the characterization of absolute continuity was first to receive attention. Bochner [3] proved that in order for $\nu$ to be absolutely continuous with respect to $\mu$, it is necessary and sufficient that $\nu$ be the limit in variation norm of a sequence of integrals of $\mu$-simple functions. Variations of Bochner's theorem, all providing characterizations of absolute continuity in terms of limits of sequences of integrals, have been obtained for a variety of settings by Darst and Green [5], Fefferman [7], and Luxemburg [9], among others. However, none of these provide an exact Radon-Nikodym derivative.

The first successful characterization of those bounded finitely additive real valued measures that have an exact Radon-Nikodym derivative with respect to $\mu$ was given by Maynard [10]. In addition to absolute continuity, the necessary and sufficient conditions he obtained require certain intricate behavior of the average range function $A_{\nu}(E)$ on the field, where $A_{\nu}(E)$ is the set of ratios $\nu(F) / \mu(F)$ for $F \subseteq E, \mu(F) \neq 0$, which involves exhaustions of $\mu$ by related classes of sets. Maynard was able to simplify his conditions for those $\mu$ which admit an exhaustive Hahn 
decomposition. He also suggested that the lack of suitable decompositions of the space was the reason that the classical Radon-Nikodym theorem did not extend to the finitely additive case.

In this paper, we introduce a separation property intermediate between disjointness and mutual singularity which generalizes Maynard's exhaustive Hahn decomposition. The two notions agree in the case where the measures to be separated are the positive and negative parts of a measure. This permits us to address the existence of exact Radon-Nikodym derivatives using separation properties without reference to the average range. The measures $\nu$ which have exact Radon-Nikodym derivatives with respect to a fixed bounded finitely additive real valued measure $\mu$ are characterized in terms of absolute continuity and the separation property. Finally, using this result, we characterize those $\mu$ for which the classical Radon-Nikodym theorem holds. Subsequently, one of the authors [8] has shown that these measures also provide a characterization of those $L_{p}$ spaces that are complete.

Throughout this paper, $\Sigma$ is a field of subsets of a nonempty set $S$ and a measure is a bounded finitely additive function mapping $\Sigma$ into $\mathbf{R}$. Notations and definitions concerning the integral considered below follow Dunford and Schwartz [6, III] except that $|\mu|$ denotes the total variation of the measure $\mu$ and $|f|$ denotes the function $|f(\cdot)|$. The notation $[f \in A]$ is shorthand for $\{s \in S \mid f(s) \in A\}$. Lattice theoretic properties may be found in [2], [4], or [12]. The symbol $\wedge$ is used for minimum both pointwise for functions and in the lattice theoretic sense for measures. More historical background of the problem is given in [10]. Most of the results of this paper were presented in [1].

2. Separation properties. Recall that measures $\eta$ and $\delta$ are said to be disjoint if $|\eta| \wedge|\delta|=0$ and mutually singular if there exists $E \in \Sigma$ such that $\eta(E)=\delta(S \sim E)=0$. It is well known that a pair of measures may be disjoint without being mutually singular.

Definition 2.1. Suppose $\eta, \delta$ and $\nu$ are measures on a field $\Sigma$. We say that $\eta$ and $\delta$ are separated if there exist increasing sequences $\left\{V_{n}\right\}$ and $\left\{W_{n}\right\}$ in $\Sigma$ such that $\lim _{n \rightarrow \infty}|\eta|\left(V_{n}\right)=|\eta|(S), \lim _{n \rightarrow \infty}|\delta|\left(W_{n}\right)=|\delta|(S)$, and $|\eta|\left(W_{n}\right)=|\delta|\left(V_{n}\right)=0$ for each $n$. In this case $\left\{V_{n}\right\}$ and $\left\{W_{n}\right\}$ is a separation of $\eta$ and $\delta$. A separation of $\nu^{+}$and $\nu^{-}$is a Hahn separation of $\nu$.

It is easy to see that the sequences in 2.1 may be chosen so that $V_{n} \cap W_{n}=\varnothing$ for each $n$ and we will do so without mention. 
It is not difficult to construct proofs and examples showing that separation is strictly weaker than mutual singularity and strictly stronger than disjointness.

If $\Sigma$ is a $\sigma$-field, then separation is equivalent to mutual singularity even if the measures are not countably additive. On the other hand even if $\Sigma$ is a $\sigma$-field and one of the measures is countably additive, disjointness does not imply separation.

REMARKS. (1) Separation is a generalization of the notion of an exhaustive Hahn decomposition due to Maynard [10] which in our terminology is a Hahn separation.

(2) Rickart [11] has considered a type of one-sided separation relative to a fixed $\sigma$-ideal in a $\sigma$-field which produces the Lebesgue decomposition when the ideal consists of the zero sets of a positive countably additive measure. This is related to separation in the following way. Given an ideal $J$ in a field $\Sigma$ and a positive measure $\mu$ one may define the measure $\mu_{J}$ by $\mu_{J}(E)=\sup \{\mu(E \cap A) \mid A \in J\}$ for $E \in \Sigma$. One may then describe our separation in terms of ideals: positive measures $\eta$ and $\delta$ are separated if and only if there are ideals $J$ and $I$ such that $J \cap I=\{\varnothing\},(\eta+\delta)_{J}=\eta$ and $(\eta+\delta)_{I}=\delta$.

That separation is a useful analogue of mutual singularity in the present setting is indicated by the theorem below.

THEOREM 2.2. Let $f$ and $g$ be integrable functions with respect to a measure $\mu$. Then $\int f d \mu$ and $\int g d \mu$ define separated measures if and only if $|f| \wedge|g|$ is a $\mu$-null function.

Proof. It suffices to prove the theorem assuming $f$ and $g$ are nonnegative functions and $\mu$ is a positive measure.

$(\rightarrow)$ Suppose that $\int f d \mu$ and $\int g d \mu$ are separated by $\left\{V_{n}\right\}$ and $\left\{W_{n}\right\}$ respectively. Note that $f \wedge g$ is $\mu$-integrable as it is $\mu$-measurable and bounded by $f$. Then for each $n \geq 1$,

$$
\begin{aligned}
\int_{S} f \wedge g d \mu & =\int_{V_{n}} f \wedge g d \mu+\int_{W_{n}} f \wedge g d \mu+\int_{V_{n}^{c} \cap W_{n}^{c}} f \wedge g d \mu \\
\leq & \int_{V_{n}} g d \mu+\int_{W_{n}} f d \mu+\int_{V_{n}^{c} \cap W_{n}^{c}} f+g d \mu \leq \int_{V_{n}^{c}} f d \mu+\int_{W_{n}^{c}} g d \mu .
\end{aligned}
$$

Since

$$
\lim _{n \rightarrow \infty} \int_{V_{n}^{c}} f d \mu=\lim _{n \rightarrow \infty} \int_{W_{n}^{c}} g d \mu=0, \quad \int_{S} f \wedge g d \mu=0 .
$$

Hence, $f \wedge g$ is a $\mu$-null function. 
$(\leftarrow)$ Now assume that $f \wedge g$ is a $\mu$-null function. It is no loss of generality to assume that $f \wedge g=0$ since $(f-f \wedge g) \wedge(g-f \wedge g)=0$, $\int f d \mu=\int(f-f \wedge g) d \mu$ and $\int g d \mu=\int(g-f \wedge g) d \mu$.

Select sequences of $\mu$-integrable simple functions $\left\{f_{n}\right\}$ and $\left\{g_{n}\right\}$ determining $f$ and $g$ respectively (following [6, III.2.17]) in such a way that $\mu^{*}\left[\left|f-f_{n}\right| \geq 1 / 2^{n}\right]<1 / 2^{n+1}$ and $\mu^{*}\left[\left|g-g_{n}\right| \geq 1 / 2^{n}\right]<1 / 2^{n+1}$ for each $n$. Choose $E_{n} \in \Sigma$ so that $\mu\left(S \sim E_{n}\right)<1 / 2^{n}$ and $S \sim E_{n} \supseteq$ $\left[\left|f-f_{n}\right| \geq 1 / 2^{n}\right] \cup\left[\left|g-g_{n}\right| \geq 1 / 2^{n}\right]$. Then $E_{n} \subseteq\left[\left|f-f_{n}\right|<1 / 2^{n}\right] \cap$ $\left[\left|g-g_{n}\right|<1 / 2^{n}\right]$. Define, for each $n$, the following sets in $\Sigma$ :

$$
\begin{aligned}
& A_{n}=E_{n} \cap\left[f_{n} \geq 1 / 2^{n}\right] \cap\left[g_{n}<1 / 2^{n}\right], \\
& B_{n}=E_{n} \cap\left[g_{n} \geq 1 / 2^{n}\right] \cap\left[f_{n}<1 / 2^{n}\right], \\
& C_{n}=E_{n} \cap\left[f_{n}<1 / 2^{n}\right] \cap\left[g_{n}<1 / 2^{n}\right], \\
& D_{n}=E_{n} \cap\left[f_{n} \geq 1 / 2^{n}\right] \cap\left[g_{n} \geq 1 / 2^{n}\right] .
\end{aligned}
$$

Now $s \in E_{n} \cap\left[f_{n} \geq 1 / 2^{n}\right]$ implies that $f(s)=f_{n}(s)-\left(f_{n}(s)-f(s)\right)$ $>1 / 2^{n}-1 / 2^{n}=0$. A similar statement holds for $g$ and $g_{n}$ replacing $f$ and $f_{n}$ respectively. Thus $A_{n} \subseteq[f>0], B_{n} \subseteq[g>0]$ and $D_{n} \subseteq[f>0]$ $\cap[g>0]$. But $f \wedge g=0$ implies that $[f>0] \cap[g>0]=\varnothing$ so that $D_{n}=\varnothing$ for each $n$.

Let $V_{n}=\bigcup_{k=1}^{n} A_{k}$ and $W_{n}=\bigcup_{k=1}^{n} B_{k}$. Then $\left\{V_{n}\right\}$ and $\left\{W_{n}\right\}$ are increasing sequences in $\Sigma$ satisfying $V_{n} \cap W_{n} \subseteq[f>0] \cap[g>0]=\varnothing$.

Next, for any positive integer $n, E_{n} \subseteq V_{n} \cup W_{n} \cup C_{n}$ so that

$$
S=V_{n} \cup W_{n} \cup C_{n} \cup\left(S \sim E_{n}\right)
$$

and

$$
\int_{S} f d \mu \leq \int_{V_{n}} f d \mu+\int_{W_{n}} f d \mu+\int_{C_{n}} f d \mu+\int_{S \sim E_{n}} f d \mu .
$$

Now $f \chi_{W_{n}}=0$ so that $\int_{W_{n}} f d \mu=0$ and since $f \chi_{C_{n}}<1 / 2^{n-1}$, $\lim _{n \rightarrow \infty} \int_{C_{n}} f d \mu=0$. Finally, $\int f d \mu \ll \mu$ and since $\mu\left(S \sim E_{n}\right)<1 / 2^{n}$, $\lim _{n \rightarrow \infty} \int_{S \sim E_{n}} f d \mu=0$. Thus $\int_{S} f d \mu=\lim _{n \rightarrow \infty} \int_{V_{n}} f d \mu$.

That $\int_{S} g d \mu=\lim _{n \rightarrow \infty} \int_{W_{n}} g d \mu$ is entirely similar, proving that $\left\{V_{n}\right\}$ and $\left\{W_{n}\right\}$ is a separation for $\int f d \mu$ and $\int g d \mu$.

Further properties of separated measures required in the next section are developed below.

LemMa 2.3. Let $\eta$ and $\delta$ be a pair of measures separated by $\left\{V_{n}\right\}$ and $\left\{W_{n}\right\}$ respectively. Then

(a) if $\eta^{\prime}$ and $\delta^{\prime}$ are measures satisfying $\eta^{\prime} \ll \eta$ and $\delta^{\prime} \ll \delta$, then $\eta^{\prime}$ and $\delta^{\prime}$ are separated by $\left\{V_{n}\right\}$ and $\left\{W_{n}\right\}$ respectively; 
(b) if $W=\bigcup_{n=1}^{\infty} W_{n}$, then $\int \chi_{W} d(\eta+\delta)$ exists and is $\delta$. Furthermore, $(\eta+\delta) *(W)=\delta(S)$.

Proof. The proof of (a) is routine. To prove (b), assume without loss of generality that $\eta$ and $\delta$ are positive measures.

The functions $\chi_{W_{n}}$ are $(\eta+\delta)$-integrable simple functions whose limit in $(\eta+\delta)$-measure is $\chi_{W}$ since for any $n$ and $\alpha>0$

$$
\begin{gathered}
(\eta+\delta)^{*}\left[\left|\chi_{W}-\chi_{W_{n}}\right|>\alpha\right]=(\eta+\delta)^{*}\left(W \sim W_{n}\right) \\
\leq(\eta+\delta)\left(S \sim\left(V_{n} \cup W_{n}\right)\right) \\
\leq \eta\left(S \sim V_{n}\right)+\delta\left(S \sim W_{n}\right) .
\end{gathered}
$$

Also, for $n>m$,

$$
\begin{aligned}
\int\left|\chi_{W_{n}}-\chi_{W_{m}}\right| d(\eta+\delta) & =\eta\left(W_{n} \sim W_{m}\right)+\delta\left(W_{n} \sim W_{m}\right) \\
& =\delta\left(W_{n} \sim W_{m}\right) \leq \delta\left(S \sim W_{m}\right)
\end{aligned}
$$

Thus, $\chi_{W}$ is $(\eta+\delta)$-integrable.

Now for $E \in \Sigma$,

$$
\begin{aligned}
\delta(E) & =\lim _{n \rightarrow \infty} \delta\left(E \cap W_{n}\right)=\lim _{n \rightarrow \infty} \int_{E} \chi_{W_{n}} d(\eta+\delta) \\
& \leq \int_{E} \chi_{W} d(\eta+\delta) \leq \lim _{n \rightarrow \infty} \int_{E} \chi_{S \sim V_{n}} d(\eta+\delta) \\
& =\lim _{n \rightarrow \infty}(\eta+\delta)\left(E \sim V_{n}\right) \\
& =\lim _{n \rightarrow \infty}(\eta+\delta)\left(E \cap W_{n}\right)+\lim _{n \rightarrow \infty}(\eta+\delta)\left(E \sim\left(V_{n} \cup W_{n}\right)\right) \\
& =\lim _{n \rightarrow \infty} \delta\left(E \cap W_{n}\right)+\lim _{n \rightarrow \infty}(\eta+\delta)\left(S \sim\left(V_{n} \cup W_{n}\right)\right)=\delta(E) .
\end{aligned}
$$

Hence, $\delta=\int \chi_{W} d(\eta+\delta)$.

Since

$$
\int_{E} \chi_{W_{n}} d(\eta+\delta) \leq(\eta+\delta)^{*}(E \cap W) \leq \int_{E} \chi_{S \sim V_{n}} d(\eta+\delta)
$$

for each $n$, the above argument also proves that $(\eta+\delta)^{*}(E \cap W)=$ $\int_{E} \chi_{W} d(\eta+\delta)=\delta(E)$ for $E \in \Sigma$. 
3. Freudenthal projections. The manner in which $\mu$ will be decomposed involves certain lattice theoretic properties of the space $b a(\Sigma)$ consisting of bounded finitely additive measures on a field $\Sigma$ which we describe briefly below and refer the reader to [2], [4], and [12] for further details.

Suppose $\eta, \lambda$ and $\mu$ are positive measures. Then $\eta$ is a component of $\mu$ if $\eta \wedge(\mu-\eta)=0$. The $\mu$-continuous $(\varepsilon-\delta$ definition) part of $\lambda$ is given by the projection of $\lambda$ onto $\mu: P_{\mu}(\lambda)=\sup \{\lambda \wedge k \mu \mid k \in \mathbf{N}\}$. The mapping $P_{\mu}$ can be extended linearly to all of $b a(\Sigma)$ and $P_{\mu}(\lambda)$ is a component of $\lambda$.

Notation. Let $\nu$ and $\mu$ be a pair of measures on $\Sigma$ satisfying $\nu \ll \mu$ and let $x$ be a real number. Then $\mu^{x+}$ and $\mu^{x-}\left(\nu^{x+}\right.$ and $\left.\nu^{x-}\right)$ denote the measures $P_{(\nu-x \mu)^{+}}(\mu)$ and $P_{(\nu-x \mu)^{-}}(\mu)\left(P_{(\nu-x \mu)^{+}}(\nu)\right.$ and $\left.P_{(\nu-x \mu)^{-}}(\nu)\right)$ respectively. Furthermore, define $\mu^{x}$ and $\nu^{x}$ by $\mu^{x}=\mu-\mu^{x+}-\mu^{x-}$ and $\nu^{x}=\nu-\nu^{x+}-\nu^{x-}$.

More precise notation would indicate that the measures defined above depend on the ordered pair $(\nu, \mu)$, but since no confusion should result where the notation is used, this cumbersome detail is omitted.

REMARK. Since $\mu$ is a weak order unit in the order complete vector lattice of $\mu$-continuous elements of $b a(\Sigma)$, the functions $\mu^{x-}$ form a Freudenthal resolution of the unity (relative to $\nu$ ). Further details may be found in [2, p. 362]. As a special case of a result given there it follows that any $\mu$-continuous $\nu$ may be written as a type of Stieltjes integral relative to the variable $x$. Projections of this type were used in [9, Th. 3.3] to obtain a Bochner type characterization of absolute continuity.

Lemma 3.1. Suppose that $\nu$ and $\mu$ are positive measures on $\Sigma, \nu \ll \mu$ and $0<x<y$. Then

(a) $\mu-P_{\nu}(\mu) \leq \mu^{x-} \leq \mu^{y-}, \lim _{z \rightarrow 0} \mu^{z-}=\mu-P_{\nu}(\mu)$ and $\lim _{z \rightarrow \infty} \mu^{z-}$ $=\mu$;

(b) $0 \leq \nu^{x-} \leq \nu^{y-}, \lim _{z \rightarrow 0} \nu^{z-}=0$ and $\lim _{z \rightarrow \infty} \nu^{z-}=\nu$;

(c) $\nu^{x-} \leq x \mu^{x-}, \nu^{x}=x \mu^{x}$ and $x \mu^{x+} \leq \nu^{x+}$;

(d) if $\mu^{y}=0$, then $\mu^{x+}-\mu^{y+}=\mu^{x+} \wedge \mu^{y-}$ and $\nu^{x+}-\nu^{y+}$ $=\nu^{x+} \wedge \nu^{v-}$

(e) $x\left(\mu^{y-} \wedge \mu^{x+}\right) \leq \nu^{y-} \wedge \nu^{x+} \leq y\left(\mu^{y-} \wedge \mu^{x+}\right)$;

(f) $\mu^{z}=\nu^{z}=0$ except for at most a countable set of real numbers $z$; and

(g) $(\nu-x \mu)^{+} \ll \mu^{x+} \ll(\nu-x \mu)^{+}$and $(\nu-x \mu)^{-} \ll \mu^{x-} \ll$ $(\nu-x \mu)^{-}$. 
Proof. We prove (e) only, as an illustration, since the other parts follow from similar properties of projections and $b a(\Sigma)$. The proof requires the following property of projections [4, p. 105]: whenever $\eta$ and $\delta$ are positive measures,

$$
P_{\eta \wedge \delta}(\cdot)=P_{\eta}(\cdot) \wedge P_{\delta}(\cdot) .
$$

Then

$$
\begin{aligned}
0 & \leq P_{(\nu-y \mu)^{-}}\left((\nu-x \mu)^{+}\right)=P_{(\nu-y \mu)^{-}}\left[P_{(\nu-x \mu)^{+}}(\nu-x \mu)\right] \\
& =P_{\left[(\nu-y \mu)^{-} \wedge(\nu-x \mu)^{+}\right]}(\nu-x \mu) \\
& =P_{(\nu-y \mu)^{-}}(\nu) \wedge P_{(\nu-x \mu)^{+}}(\nu)-x\left[P_{(\nu-y \mu)^{-}}(\mu) \wedge P_{(\nu-x \mu)^{+}}(\mu)\right] \\
& =\nu^{y-} \wedge \nu^{x+}-x\left[\mu^{y-} \wedge \mu^{x+}\right]
\end{aligned}
$$

and therefore $x\left(\mu^{y-} \wedge \mu^{x+}\right) \leq \nu^{y-} \wedge \nu^{x+}$. The other inequality is obtained similarly.

4. An exact Radon-Nikodym theorem. We will need the following technical lemma.

LEMMA 4.1. Let $\nu$ and $\mu$ be positive measures on $\Sigma$ satisfying

(a) $\nu \ll \mu$ and

(b) there is a sequence $\left\{x_{n}\right\}_{n=1}^{\infty}$ of positive real numbers, such that $\nu-x_{n} \mu$ has a Hahn separation for each $n$. Then sequences $\left\{V_{l}^{n}\right\}_{l}$ and $\left\{W_{i}^{n}\right\}_{i}$ separating $\left(\nu-x_{n} \mu\right)^{-}$and $\left(\nu-x_{n} \mu\right)^{+}$respectively can be chosen so that whenever $x_{m}<x_{n}, \cup_{l=1}^{\infty} W_{l}^{n} \subseteq \bigcup_{i=1}^{\infty} W_{i}^{m}$.

The sequences can be constructed inductively using elementary set theoretic operations. The details are left to the reader.

We are now ready to prove the Radon-Nikodym theorem for bounded finitely additive measures.

THEOREM 4.2. Let $\nu$ be a measure and $\mu$ a positive measure on a field $\Sigma$. Then there exists a $\mu$-integrable function $f$ such that $\nu(E)=\int_{E} f d \mu$ for all $E \in \Sigma$ if and only if

(a) $\nu \ll \mu$ and

(b) $\nu-x \mu$ has a Hahn separation for each $x \neq 0$.

Proof. $(\rightarrow)$ Let $\nu=\int f d \mu$ for a $\mu$-integrable function $f$. Then $\nu \ll \mu$ is a known result [6]. 
Now, for $x \neq 0, \quad \nu-x \mu=\int f d \mu-\int x d \mu=\int(f-x) d \mu$. Hence, $(\nu-x \mu)^{+}=\int(f-x)^{+} d \mu$ and $(\nu-x \mu)^{-}=\int(f-x)^{-} d \mu$. Since $(f-x)^{+} \wedge(f-x)^{-}=0,(\nu-x \mu)^{+}$and $(\nu-x \mu)^{-}$are separated by Theorem 2.2.

$(\leftarrow)$ Suppose that $\nu \ll \mu$ and $\nu-x \mu$ has a Hahn separation for each $x \neq 0$. For the time being, assume that $\nu$ is positive.

Let $\left\{x_{n}\right\}_{n=1}^{\infty}$ be a dense sequence in $\mathbf{R}^{+}$for which $\mu^{x_{n}}=\nu^{x_{n}}=0$ for every $n$. As in Lemma 4.1, obtain separations $\left\{V_{i}^{n}\right\}$ and $\left\{W_{i}^{n}\right\}$ for $\left(\nu-x_{n} \mu\right)^{-}$and $\left(\nu-x_{n} \mu\right)^{+}$such that $\bigcup_{i=1}^{\infty} W_{i}^{n} \subseteq \bigcup_{i=1}^{\infty} W_{i}^{m}$ whenever $x_{m}$ $<x_{n}$. Since $\mu^{x_{n}-} \ll\left(\nu-x_{n} \mu\right)^{-}$and $\mu^{x_{n}+} \ll\left(\nu-x_{n} \mu\right)^{+},\left\{V_{i}^{n}\right\}$ and $\left\{W_{i}^{n}\right\}$ is a separation for $\mu^{x_{n}-}$ and $\mu^{x_{n}+}$ respectively by Lemma 2.3. Let $x_{0}=0$, $W_{0}=S$ and, for each $n \geq 1$, set $W_{n}=\bigcup_{i=1}^{\infty} W_{i}^{n}$. Then $W_{n} \subseteq W_{n}$ whenever $x_{m}<x_{n}$. Define functions $f_{n}$ for each $n \geq 1$ and $f$ by $f_{n}(s)=\max \left\{x_{k}\right\}$ $\left.s \in W_{k}, k \leq n\right\}$ and $f(s)=\sup \left\{x_{k} \mid s \in W_{k}\right\}$. Note that $f_{n}$ increases with $n$ and $0 \leq f_{n} \leq f$.

$f_{n}$ is $\mu$-integrable and $\int f_{n} d \mu \leq \nu$. Fix $n \geq 1$ and assume without loss of generality that $x_{k} \leq x_{k+1}$ for $k=1,2, \ldots, n-1$. Then

$$
f_{n}=\sum_{k=1}^{n-1} x_{k} \chi_{W_{k}-W_{k+1}}+x_{n} \chi_{W_{n}}=\sum_{k=1}^{n-1} x_{k}\left(\chi_{W_{k}}-\chi_{W_{k+1}}\right)+x_{n} \chi_{W_{n}},
$$

which, being a linear combination of $\mu$-integrable functions (Lemma 2.3) is itself $\mu$-integrable.

Since $\int_{E} \chi_{W_{k}} d \mu=\mu^{x_{k}+}(E)$ by Lemma 2.3

$$
\begin{aligned}
\int f_{n} d \mu & =\sum_{k=1}^{n-1} x_{k}\left(\mu^{x_{k}+}-\mu^{x_{k+1}+}\right)+x_{n} \mu^{x_{n}+} \\
& \leq \sum_{k=1}^{n-1}\left(\nu^{x_{k}+}-\nu^{x_{k+1}+}\right)+\nu^{x_{n}+}=\nu^{x_{1}+} \leq \nu .
\end{aligned}
$$

$f$ is $\mu$-integrable and $\int f d \mu=\nu$. Since $\int f_{n} d \mu \leq \nu \ll \mu$, the indefinite integrals $\int f_{n} d \mu$ are uniformly absolutely continuous with respect to $\mu$ and since $\mu$ is bounded, it remains to show that $f_{n} \rightarrow f$ in $\mu$-measure to obtain the $\mu$-integrability of $f$. The equality $\int f d \mu=\nu$ will then follow once the convergence $\int f_{n} d \mu \rightarrow \nu$ in variation is established.

Let $\varepsilon>0$ and choose $N$ so that $\mu^{x_{N}+}(S)<\varepsilon$ and $\nu^{x_{N}+}(S)<\varepsilon$. Select $M>N$ such that $\left\{x_{1}, x_{2}, \ldots, x_{M}\right\}$ intersects every interval of length $\varepsilon$ contained in $\left[0, x_{N}\right]$. Let $G=\left\{x_{k} \mid x_{k} \leq x_{N}\right.$ and $\left.0 \leq k \leq M\right\}$. Relabel the elements of $G$ using $\left\{y_{0}, y_{1}, \ldots, y_{p}\right\}$ where $x_{0}=0=y_{0}<y_{1}<\cdots<y_{p}$ $=x_{N}$ and set $B_{k}=W_{j}$ if $y_{k}=x_{j}$. Then

(i) $\mu^{y_{p}+}(S)<\varepsilon$ and $\nu^{y_{p}+}(S)<\varepsilon$,

(ii) $0<y_{k+1}-y_{k}<\varepsilon$ for $k=0,1, \ldots, p-1$, and

(iii) $B_{k} \supseteq B_{k+1}$ for $k=0,1, \ldots, p-1$. 
Now let $n>M$. For any $s \in B_{0} \sim B_{p}$, there is an $i, 0 \leq i<p$, such that $s \in B_{l} \sim B_{i+1}$. Then $y_{l} \leq f_{M}(s) \leq f_{n}(s) \leq f(s) \leq y_{i+1}$; hence $0 \leq$ $f(s)-f_{n}(s) \leq \varepsilon$. Therefore $\left[\left|f-f_{n}\right| \geq \varepsilon\right] \subseteq B_{p}$ so that $\mu^{*}\left[\left|f-f_{n}\right| \geq \varepsilon\right] \leq$ $\mu^{*}\left(B_{p}\right)=\left(\mu^{y_{p}-}+\mu^{y_{p}+}\right)^{*}\left(B_{p}\right)=\mu^{y_{p}+}(S)<\varepsilon$ by Lemma 3.1 and $\mu^{y_{p}}=0$. Thus $f_{n} \rightarrow f$ in $\mu$-measure, proving the $\mu$-integrability of $f$.

Again, let $n>M$ in the set-up above. For $k=1,2, \ldots, p-1$, let $\mu_{k}=\mu^{v_{k}+}-\mu^{y_{k+1}+}, \quad \nu_{k}=\nu^{y_{k}+}-\nu^{y_{k+1}+}$ and $A_{k}=B_{k} \sim B_{k+1}=1$. By Lemma 3.1, $y_{k} \mu_{k} \leq \nu_{k} \leq y_{k+1} \mu_{k}, k=1,2, \ldots, p-1$. Note that $\nu=$ $\sum_{k=1}^{p-1} \nu_{k}+\nu^{v_{p}+}+\nu^{y_{1}-}$ and similarly for $\mu$. Also,

$$
\int \chi_{A_{k}} d \mu=\int \chi_{B_{k}} d \mu-\int \chi_{B_{k+1}} d \mu=\mu^{y_{k}+}-\mu^{y_{k+1}+}=\mu_{k} .
$$

Put $h=\sum_{k=1}^{p-1} y_{k} \chi_{A_{k}}+y_{p} \chi_{B_{p}}$. Thus $h$ is $\mu$-integrable and $0 \leq h \leq f_{M}$ $\leq f_{n}$. Furthermore, $\int h d \mu=\sum_{k=1}^{p-1} y_{k} \mu_{k}+y_{p} \mu^{y_{p}+}$. For any $E \in \Sigma$,

$$
\begin{aligned}
0 & \leq\left|\nu-\int f_{n} d \mu\right|(S)=\nu(S)-\int_{S} f_{n} d \mu \leq \nu(S)-\int_{S} h d \mu \\
& \leq \sum_{k=1}^{p-1} \nu_{k}(S)+\nu^{y_{p}+}(S)+\nu^{y_{1}}-(S)-\sum_{k=1}^{p-1} y_{k} \mu_{k}(S) \\
& \leq \sum_{k=1}^{p-1} y_{k+1} \mu_{k}(S)-\sum_{k=1}^{p-1} y_{k} \mu_{k}(S)+\nu^{y_{p}}(S)+\nu^{y_{1}}-(S) \\
& <\sum_{k=1}^{p-1}\left(y_{k+1}-y_{k}\right) \mu_{k}(S)+\varepsilon+y_{1} \mu^{y_{1}}-(S) \\
& <\varepsilon \sum_{k=1}^{p-1} \mu_{k}(S)+\varepsilon+\varepsilon \mu^{y_{1}-}(S) \\
& <\varepsilon \mu(S)+\varepsilon .
\end{aligned}
$$

Thus $\int f_{n} d \mu \rightarrow \nu$ in variation, completing the proof when $\nu$ is a positive measure.

Now let $\nu$ be a signed measure such that $\nu \ll \mu$ and $\nu-x \mu$ has a Hahn separation for each $x \neq 0$. For $x>0$ fixed, $\nu-x \mu=\nu^{+}-$ $x \mu-\nu^{-}=\left(\nu^{+}-x \mu\right)^{+}-\left[\left(\nu^{+}-x \mu\right)^{-}+\nu^{-}\right]$. Since $0 \leq\left(\nu^{+}-x \mu\right)^{+} \leq \nu^{+}$, $\left(\nu^{+}-x \mu\right)^{+} \wedge \nu^{-}=0$ so that $\left(\nu^{+}-x \mu\right)^{+} \wedge\left[\left(\nu^{+}-x \mu\right)^{-}+\nu^{-}\right]=0$. By the uniqueness of the Jordan decomposition, $(\nu-x \mu)^{+}=\left(\nu^{+}-x \mu\right)^{+}$and $(\nu-x \mu)^{-}=\left(\nu^{+}-x \mu\right)^{-}+\nu^{-}$. These by assumption are separated and hence $\left(\nu^{+}-x \mu\right)^{+}$and $\left(\nu^{+}-x \mu\right)^{-}$are separated. Similarly $\nu^{+}-\nu^{-}+x \mu$ has a Hahn separation for each $x>0$ which leads to a separation of $\left(\nu^{-}-x \mu\right)^{+}$and $\left(\nu^{-}-x \mu\right)^{-}$. By the proof above, $\nu^{+}$and $\nu^{-}$can be represented as indefinite integrals with respect to $\mu$ whose difference is then $\nu$. 
COROLlaRy 4.3. Let $\nu$ be a measure and $\mu$ a positive measure on a $\sigma$-field $\Sigma$. Then there exists a $\mu$-integrable function $f$ such that $\nu(E)=\int_{E} f d \mu$ for all $E \in \Sigma$ if and only if

(a) $\nu \ll \mu$,

(b) $\nu-x \mu$ has a Hahn decomposition for each $x \neq 0$.

Thus, in the case of $\sigma$-fields, Maynard's suggestion that the classical Radon-Nikodym fails to hold in the finitely additive case because of the lack of Hahn decompositions is confirmed.

REMARK. If, in addition to $\Sigma$ being a $\sigma$-field, $|\nu| \leq k \mu$ for some $k \in \mathbf{R}$, then the representing function $f$ can be obtained as a uniform limit of simple functions on $\Sigma$. This leads to the following observation, similar to one given in $[9,48]: \nu$ may be represented by a uniform limit of simple functions on $\Sigma$ if and only if $\nu-x \mu$ has a Hahn decomposition for each $x \neq 0$. The following example shows that this does not hold on arbitrary fields. Let $\Sigma$ be the field of finite subsets of $\mathbf{N}$ and their complements. Let $a_{n}$ be the $0-1$ valued measure at $n$ for each $n$. Let

$$
\mu=\sum_{n=1}^{\infty} \frac{1}{2^{n}} a_{n} \text { and } \quad \nu=\sum_{n=1}^{\infty} \frac{1}{2^{n}}\left(1+\frac{(-1)^{n}}{n}\right) a_{n} .
$$

Then $\nu=\int f d \mu$ where $f(k)=1+(-1)^{k} / k$ for every $k$, while $\nu-1 \cdot \mu$ does not have a Hahn decomposition.

The conclusions in Theorem 4.2 remain valid when $\mu$ is a signed measure which has a Hahn separation, but when no such separation exists, condition (b) is not necessary for the existence of $f$. Take for example a signed measure $\mu$ which does not have a Hahn separation and let $\nu=\mu$. Then $\nu=\int 1 d \mu$, yet $\nu-x \mu$ has no Hahn separation for $x \neq 1$. The modification required for the case where $\mu$ is a signed measure is contained in the theorem below. This makes use of the signed projection onto $\mu: P_{[\mu]}(\lambda)=P_{\mu^{+}}(\lambda)-P_{\mu^{-}}(\lambda)$. Note that $P_{[\mu]}(\nu-x \mu)=P_{[\mu]}(\nu)-x|\mu|$.

THEOREM 4.4. Let $\nu$ and $\mu$ be measures on a field $\Sigma$. Then there exists a $\mu$-integrable function $f$ such that $\nu(E)=\int_{E} f d \mu$ for all $E \in \Sigma$ if and only if

(a) $\nu \ll \mu$ and

(b) $P_{[\mu]}(\nu-x \mu)$ has a Hahn separation for each $x \neq 0$.

Proof. The identities $P_{|\mu|}=P_{[\mu]}^{2}$ and $P_{[\mu]}\left(\int f d|\mu|\right)=\int f d \mu$ together with an application of Theorem 4.2 to $P_{[\mu]}(\nu)$ and $|\mu|$ produce the conclusion. 
We can now characterize the measures $\mu$ for which $\nu \ll \mu$ is equivalent to $\nu=\int f d \mu$ for some $\mu$-integrable function $f$.

COROLlaRY 4.5. Let $\mu$ be a measure on a field (respectively, $\sigma$-field) $\Sigma$. Then a necessary and sufficient condition on $\mu$ for every measure $\nu \ll \mu$ to take the form $\nu=\int f d \mu$ for some $\mu$-integrable function $f$ is that each pair of disjoint components of $|\mu|$ be separated (respectively, mutually singular).

Proof. We first prove the theorem assuming $\mu \geq 0$. Suppose each pair of disjoint components of $\mu$ are separated. Then for $\nu \ll \mu$ and $x \neq 0$, $\mu^{x+}$ and $\mu^{x-}$ are disjoint components of $\mu$ and therefore are separated. By Lemmas 2.3 and $3.1 \mathrm{~g}$, the associated separation provides a Hahn separation for $\nu-x \mu$ and Theorem 4.2 applies.

On the other hand, if $\mu$ has disjoint components $\eta$ and $\delta$ which are not separated, then $\delta$ and $\mu-\delta$ cannot be separated. Set $\nu=\mu+2 \delta$. Then $\nu \ll \mu$, yet $\nu-2 \mu=\delta-(\mu-\delta)$ does not have a Hahn separation.

For the case where $\mu$ is a signed measure, we use the fact that if there exists a $\mu$-integrable function $h$ such that $|\mu|=\int h d \mu$, then $\mu=\int h d|\mu|$ and conversely. If such a function $h$ exists, we may assume it is bounded, in which case, whenever $\nu \ll \mu$, there exists a $\mu$-integrable function $f$ such that $\nu=\int f d \mu$ if and only if there exists a $\mu$-integrable function $g$ such that $\nu=\int g d|\mu|(g=f h)$.

Thus, it remains to show that either condition implies the existence of a function $h$ such that $\int h d \mu=|\mu|$ or $\int h d|\mu|=\mu$.

Suppose that disjoint components of $|\mu|$ can be separated. Then by the proof above and $\mu \ll|\mu|, \mu=\int h d|\mu|$ for some $\mu$-integrable function $h$.

Next, assume that each measure $\nu \ll \mu$ can be expressed as an indefinite integral with respect to $\mu$. Then $|\mu| \ll \mu$ so $|\mu|=\int h d \mu$ for some $\mu$-integrable function $h$. This completes the proof.

Finally, we note that the classical Radon-Nikodym theorem for real valued countably additive measures on a $\sigma$-field is an immediate consequence of these results since in that setting all of the measures in question have Hahn decompositions and hence condition (b) is trivially satisfied.

\section{REFERENCES}

[1] Wayne C. Bell and John W. Hagood, Separation properties of finitely additive measures and the Radon-Nikodym theorem, Proc. Confer. on Measure Theory and its Application, N. I1l. Univ., Oct. (1980), 161-163.

[2] Garrett Birkhoff, Lattice Theory, Amer. Math. Soc. Colloquium Publications, Vol. 25 (3rd edition) 1967. 
[3] S. Bochner, Additive set functions on groups, Ann. of Math., 40 (1939), 769-799.

[4] R. Cristescu, Ordered Vector Spaces and Linear Operators, Abacus Press, Turnbridge Wells, Kent, England, 1976.

[5] R. B. Darst and Euline Green, On a Radon-Nikodym theorem for finitely additive set functions, Pacific J. Math., 27 (1968), 255-259.

[6] N. Dunford and J. T. Schwartz, Linear Operators, Interscience, New York, 1958.

[7] C. Fefferman, $A$ Radon-Nikodym theorem for finitely additive set functions, Pacific J. Math., 23 (1967), 35-45.

[8] John W. Hagood, A Radon-Nikodym theorem and $L_{p}$ completeness for finitely additive vector measures, J. Math. Anal. Appl., 113 (1986), 266-279.

[9] W. A. J. Luxemburg, Orthomorphisms and the Radon-Nikodym theorem revisited, From A to Z, Mathematical Centre Tracts \#149, Amsterdam (1982), 39-50.

[10] Hugh B. Maynard, A Radon-Nikodym theorem for finitely additive bounded measures, Pacific J. Math., 83 (2) (1979), 401-413.

[11] C. E. Rickart, Decomposition of additive set functions, Duke Math. J., (1943), 653-665.

[12] H. H. Schaeffer, Banach Lattices and Positive Operators, Springer Verlag, Heidelberg, Berlin, 1974.

Received September 29, 1986.

MurRay State University

MURRAY, KY 42071

AND

NORTHERN ARIZONA UNIVERSITY

FlagstaFF, AZ 86011 


\section{PACIFIC JOURNAL OF MATHEMATICS EDITORS}

\author{
V. S. VARADARAJAN \\ (Managing Editor) \\ University of California \\ Los Angeles, CA 90024 \\ HERBERT ClEMENS \\ University of Utah \\ Salt Lake City, UT 84112 \\ R. FINN \\ Stanford University \\ Stanford, CA 94305
}

\author{
HERMANN FLASCHKA \\ University of Arizona \\ Tucson, AZ 85721
}

RAMESh A. GANGolli

University of Washington Seattle, WA 98195

VAUGHAN F. R. JONES

University of California

Berkeley, CA 94720

\author{
ROBION KIRBY \\ University of California \\ Berkeley, CA 94720
}

C. C. MOORE

University of California

Berkeley, CA 94720

HAROLD STARK

University of California, San Diego

La Jolla, CA 92093

\section{ASSOCIATE EDITORS}
R. AREnS
E. F. BECKENBACH
B. H. NEUMANN
F. WOLF
K. YOSHIDA

(1906-1982)

\section{SUPPORTING INSTITUTIONS}

\begin{abstract}
UNIVERSITY OF ARIZONA
UNIVERSITY OF BRITISH COLUMBIA

CALIFORNIA INSTITUTE OF TECHNOLOGY

UNIVERSITY OF CALIFORNIA

MONTANA STATE UNIVERSITY

UNIVERSITY OF NEVADA, RENO

NEW MEXICO STATE UNIVERSITY

OREGON STATE UNIVERSITY
\end{abstract}

\author{
UNIVERSITY OF OREGON \\ UNIVERSITY OF SOUTHERN CALIFORNIA \\ STANFORD UNIVERSITY \\ UNIVERSITY OF HAWAII \\ UNIVERSITY OF TOKYO \\ UNIVERSITY OF UTAH \\ WASHINGTON STATE UNIVERSITY \\ UNIVERSITY OF WASHINGTON
}

The Supporting Institutions listed above contribute to the cost of publication of this Journal, but they are not owners or publishers and have no responsibility for its content or policies.

Mathematical papers intended for publication in the Pacific Journal of Mathematics should be in typed form or offset-reproduced (not dittoed), double spaced with large margins. Please do not use built up fractions in the text of the manuscript. However, you may use them in the displayed equations. Underline Greek letters in red, German in green, and script in blue. The first paragraph must be capable of being used separately as a synopsis of the entire paper. In particular it should contain no bibliographic references. Please propose a heading for the odd numbered pages of less than 35 characters. Manuscripts, in triplicate, may be sent to any one of the editors. Please classify according to the scheme of Math. Reviews, Index to Vol. 39. Supply name and address of author to whom proofs should be sent. All other communications should be addressed to the managing editor, or Elaine Barth, University of California, Los Angeles, California 90024.

There are page-charges associated with articles appearing in the Pacific Journal of Mathematics. These charges are expected to be paid by the author's University, Government Agency or Company. If the author or authors do not have access to such Institutional support these charges are waived. Single authors will receive $\mathbf{5 0}$ free reprints; joint authors will receive a total of $\mathbf{1 0 0}$ free reprints. Additional copies may be obtained at cost in multiples of 50 .

The Pacific Journal of Mathematics is issued monthly as of January 1966. Regular subscription rate: $\$ 190.00$ a year (5 Vols., 10 issues). Special rate: $\$ 95.00$ a year to individual members of supporting institutions.

Subscriptions, orders for numbers issued in the last three calendar years, and changes of address should be sent to Pacific Journal of Mathematics, P.O. Box 969, Carmel Valley, CA 93924, U.S.A. Old back numbers obtainable from Kraus Periodicals Co., Route 100, Millwood, NY 10546.

The Pacific Journal of Mathematics at P.O. Box 969, Carmel Valley, CA 93924 (ISSN 0030-8730) publishes 5 volumes per year. Application to mail at Second-class postage rates is pending at Carmel Valley, California, and additional mailing offices. Postmaster: send address changes to Pacific Journal of Mathematics, P.O. Box 969, Carmel Valley, CA 93924.

PUBLISHED BY PACIFIC JOURNAL OF MATHEMATICS, A NON-PROFIT CORPORATION Copyright (C) 1988 by Pacific Journal of Mathematics 


\section{Pacific Journal of Mathematics}

\section{Vol. 131, No. 2 December, 1988}

Selman Akbulut and Henry Churchill King, Polynomial equations of immersed surfaces .................................... 209

Alberto Baider and Richard C. Churchill, The Campbell-Hausdorff group and a polar decomposition of graded algebra automorphisms ........2 219

Wayne C. Bell and John William Hagood, Separation properties and exact

Radon-Nikodým derivatives for bounded finitely additive measures . . . 237

Dennis J. Garity, James P. Henderson and David G. Wright, Menger

spaces and inverse limits ...............................249

B. Brent Gordon, Algebraically defined subspaces in the cohomology of a

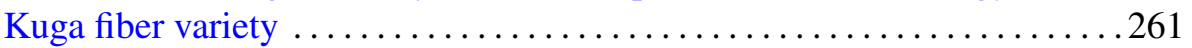

Jeffrey A. Hogan, Weighted norm inequalities for the Fourier transform on connected locally compact groups ........................... 277

Guojun Liao, A study of regularity problem of harmonic maps ..........291

Chin-pi Lu, Modules satisfying ACC on a certain type of colons ......... 303

Kunio Murasugi, Jones polynomials of periodic links

Hans Schoutens, Approximation properties for some non-Noetherian local

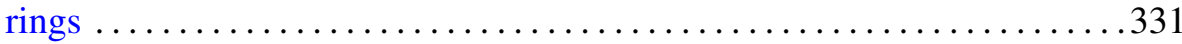

Peter Sjögren, Convergence for the square root of the Poisson kernel ...... 361 Alexandru Ion Suciu, The oriented homotopy type of spun 3-manifolds .393 